\title{
A phase I study assessing the feasibility and safety of intraductal pegylated liposomal doxorubicin (PLD) in women awaiting mastectomy
}

\author{
V Stearns*, L Jacobs, N Khouri, S Jeter, P Powers, K Shahverdi, R Brown, \\ M Rudek, E Gabrielson, Z Zhang, T Tsangaris and S Sukumar
}

Address: Breast Cancer Program, Sidney Kimmel Comprehensive Cancer Center, Johns Hopkins School of Medicine, Baltimore, MD, USA

Email: V Stearns* - Vstearn1@jhmi.edu

* Corresponding author

from 6th International Symposium on the Intraductal Approach to Breast Cancer Santa Monica, CA, USA. |9-2| February 2009

Published: 24 July 2009

BMC Proceedings 2009, 3(Suppl 5):S28 doi:10.1 I86/1753-656I-3-S5-S28

This abstract is available from: http://www.biomedcentral.com/I753-656I/3/S5/S28

(c) 2009 Stearns et al; licensee BioMed Central Ltd.

\section{Background}

Our preclinical data have demonstrated that intraductal administration of PLD decreases tumor volume, prevents the development of new lesions, and eradicates pre-malignant disease. We initiated a phase I study to determine the feasibility, safety, and maximum tolerated dose of PLD administered to women awaiting mastectomy.

\section{Methods}

Women 18 or older with a known breast cancer awaiting a mastectomy were eligible. Neoadjuvant chemotherapy was allowed. Women with T4 features, prior breast irradiation, or procedures that in the opinion of the investigator may have altered the breast ductal system were excluded. Participants underwent nipple aspiration and ductal cannulation using a dose escalation schema. The first 3 women received $5 \mathrm{~mL}$ intraductal dextrose only. We determined serial doxorubicin and doxorubicinol concentrations in plasma and nipple aspirate fluid using LC/MS/ MS. We injected blue dye into the treated duct just prior to mastectomy and obtained tissue for pharmacokinetic and biomarker analysis.

\section{Results}

From 02/06 to 09/08, 14 women entered the study, and 12 underwent study procedures successfully. We completed all dose levels up to $10 \mathrm{mg}$ PLD per one duct with- out serious adverse events or surgical delays. Pharmacokinetic and representative histopathological data will be presented.

\section{Conclusion}

Intraductal administration of PLD is feasible and can be safely administered both in women with and without prior chemotherapy awaiting mastectomy. Studies to evaluate other agents administered to one or more ducts are required. 